\title{
OPEN Efficacy of loading dose colistin versus carbapenems for treatment of extended spectrum beta lactamase producing Enterobacteriaceae
}

\author{
Wasan Katip ${ }^{1,2 \bowtie}$, Jukapun Yoodee ${ }^{1}$, Suriyon Uitrakul ${ }^{3} \&$ Peninnah Oberdorfer ${ }^{2,4}$
}

Colistin provides in vitro activity against numerous ESBL-producing and carbapenem-resistant bacteria. However, clinical information with respect to its utilization in infection caused by ESBL producers is limited. The aim of this study was a comparison of mortality rates of loading dose (LD) colistin and carbapenems as definitive therapies in a cohort of patients with infections caused by ESBL-producing Escherichia coli and Klebsiella pneumoniae. A retrospective cohort study in 396 patients with ESBL-producing E.coli and $K$.pneumoniae infection at a university-affiliated hospital was conducted between 1 January 2005 and 30 June 2015 to compare outcomes of infected patients who received LD colistin (95 patients) with carbapenems (301 patients). The three primary outcomes were 30-day mortality, clinical response and microbiological response. The most common infection types were urinary tract infection $(49.49 \%)$, followed by pneumonia $(40.66 \%)$, bacteremia $(13.64 \%)$, skin and soft tissue infections (4.80\%) and intra-abdominal infection (3.03\%). LD colistin group provided higher 30-day mortality when compared with carbapenems group (HR 7.97; $95 \% \mathrm{Cl} 3.68$ to $17.25 ; P=0.001$ ). LD colistin was also independently associated with clinical failure (HR 4.30; 95\% Cl 1.93 to 9.57; $P=0.001$ ) and bacteriological failure (HR $9.49 ; 95 \% \mathrm{Cl} 3.76$ to $23.96 ; P=0.001$ ) when compared with those who received carbapenems. LD colistin treatment was associated with poorer outcomes, i.e. mortality rate, clinical response and microbiological response. Moreover, when adjusted confounding factors, LD colistin was still less effective than carbapenems. It should be noted that, however, the use of Vitek- 2 to assess colistin susceptibility could provide inaccurate results. Also, the difference in baseline characteristics could still remain in retrospective study although compensation by hazard ratio adjustment was performed. Therefore, clinical utilization of $L D$ colistin should be recommended as an alternative for treatment ESBL-producing Enterobacteriaceae only in the circumstances where carbapenems cannot be utilized, but this recommendation must be considered carefully.

Extended-spectrum $\beta$-lactamase (ESBL)-producing Enterobacteriaceae has emerged worldwide and has become important nosocomial infection. A retrospective cohort study that was conducted in Sa Kaeo and Nakhon Phanom provinces, Thailand, reported that the overall mortality rate of bloodstream infections due to ESBLproducing Enterobacteriaceae from 2008 and 2014 was $20.0 \%{ }^{1}$.

ESBL production limits the number of drugs that can be used for an effective treatment of an infection ${ }^{2}$. However, the effectiveness of antimicrobial agents for the treatment of ESBL-producing Enterobacteriaceae is controversial $^{2,3}$. In a multinational study of 85 patients with ESBL-producing Klebsiella pneumoniae bacteremia, carbapenems provided the lowest 14 -day mortality ${ }^{4}$. Moreover, a meta-analysis from non-randomized studies compared mortality rates between carbapenems and alternative antibiotics for the treatment of ESBLpositive Enterobacteriaceae bacteraemia, and reported lower mortality when using carbapenems than alternative

\footnotetext{
${ }^{1}$ Department of Pharmaceutical Care, Faculty of Pharmacy, Chiang Mai University, Chiang Mai 50200, Thailand. ${ }^{2}$ Epidemiology Research Group of Infectious Disease (ERGID), Chiang Mai University, Chiang Mai 50200, Thailand. ${ }^{3}$ Department of Pharmaceutical Care, School of Pharmacy, Walailak University, Nakhon Si Thammarat 80160, Thailand. "Division of Infectious Diseases, Department of Pediatrics, Faculty of Medicine, Chiang Mai University, Chiang Mai 50200,Thailand. ${ }^{\circledR}$ email: wasankatip@gmail.com
} 


\begin{tabular}{|c|c|c|c|}
\hline \multirow[b]{2}{*}{ Characteristic } & \multicolumn{3}{|c|}{ Definitive therapy cohort } \\
\hline & $\begin{array}{l}\text { LD colistin } \\
(\mathrm{n}=95)\end{array}$ & $\begin{array}{l}\text { Carbapenems } \\
(\mathrm{n}=301)\end{array}$ & $p$ value \\
\hline Age $(y r)($ mean $\pm S D)$ & $65.04 \pm 16.60$ & $62.96 \pm 19.82$ & 0.357 \\
\hline Female & $61(64.21)$ & $148(49.17)$ & $0.013^{\mathrm{a}}$ \\
\hline ICU admission & $64(67.37)$ & $44(14.62)$ & $0.001^{\mathrm{a}}$ \\
\hline Mechanical ventilation & $75(78.95)$ & $251(83.39)$ & 0.355 \\
\hline Duration of hospitalization, median (min-max) & $34(4-157)$ & $12(5-58)$ & $0.001^{\mathrm{a}}$ \\
\hline Appropriate antibiotic treatment & $84(88.42)$ & $295(98.01)$ & $0.001^{\mathrm{a}}$ \\
\hline Charlson score $($ mean \pm SD) & $2.73 \pm 2.35$ & $4.43 \pm 2.22$ & $0.001^{\mathrm{a}}$ \\
\hline \multicolumn{4}{|l|}{ Underlying diseases } \\
\hline Cerebrovascular disease & $29(30.85)$ & $175(58.14)$ & $0.001^{\mathrm{a}}$ \\
\hline Diabetes mellitus & $20(21.05)$ & $92(30.56)$ & $0.089^{\mathrm{a}}$ \\
\hline Chronic kidney disease & $21(22.34)$ & $60(20.00)$ & 0.661 \\
\hline Malignancy & $25(26.60)$ & $93(30.90)$ & 0.519 \\
\hline COPD & $10(10.53)$ & $38(12.62)$ & 0.719 \\
\hline Liver disease & $9(9.57)$ & $17(5.65)$ & 0.231 \\
\hline \multicolumn{4}{|l|}{ Infection type } \\
\hline Pneumonia & $45(47.37)$ & $116(38.54)$ & $0.150^{\mathrm{a}}$ \\
\hline Urinary tract infection & $31(32.63)$ & $165(54.82)$ & $0.001^{\mathrm{a}}$ \\
\hline Bloodstream infection & $41(43.16)$ & $13(4.32)$ & $0.001^{\mathrm{a}}$ \\
\hline Skin and soft-tissues, surgical sites, joints, and bones & $13(13.68)$ & $6(1.99)$ & $0.001^{\mathrm{a}}$ \\
\hline Intra-abdominal infection & $5(5.26)$ & $7(2.33)$ & $0.169^{\mathrm{a}}$ \\
\hline Duration of treatment $($ mean $\pm S D)$ & $8.47 \pm 4.86$ & $11.17 \pm 3.95$ & $0.001^{\mathrm{a}}$ \\
\hline \multicolumn{4}{|l|}{ Pathogen causing infection } \\
\hline K. pneumoniae ESBL & $45(47.37)$ & $147(48.84)$ & 0.815 \\
\hline E.coli $\mathrm{ESBL}$ & $55(57.89)$ & $154(51.16)$ & 0.289 \\
\hline Nephrotoxicity & $46(48.42)$ & $0(0.00)$ & $0.001^{\mathrm{a}}$ \\
\hline
\end{tabular}

Table 1. Characteristics of patient infection caused by ESBL-producing E. coli and K. pneumoniae. ${ }^{\text {a Variable }}$ used to adjustment for treatment with LD colistin.

antibiotics for definitive and empirical treatment ${ }^{5}$. Therefore, carbapenems have become the standard treatment of ESBL-producing Enterobacteriaceae ${ }^{2,3,5}$.

Colistin or polymyxin $\mathrm{E}$ is an old antimicrobial agent which was discovered from Bacillus polymyxa, subspecies Colistinus Koyama. In 1949, this agent was used in the form of colistimethate sodium to treat infections caused by extensively drug-resistant Gram-negative bacteria. (XDR-GNB) ${ }^{6,7}$. Colistin is one of antibiotics that exhibited excellent in vitro efficacy against ESBL-producing Enterobacteriaceae ${ }^{8}$. There has been a few case reports of successful use of polymyxins to treat ESBL-associated infections, and to date only a few clinical studies concerning the efficacy of colistin for treatment of infections with ESBL-producing Escherichia coli and $K$. pneumoniae ${ }^{9,10}$. Therefore, the aim of this study was to evaluate clinical efficacy of loading dose (LD) colistin compared with carbapenems for treatment ESBL-producing Escherichia coli and Klebsiella pneumoniae.

\section{Results}

A total of 396 cases were included; 95 patients received with LD colistin and 301 patients with carbapenems (241 patients with meropenem, 40 with ertapenem and 20 with imipenem). Characteristics of patient infection caused by ESBL-producing Enterobacteriaceae are shown in Table 1. The minimum inhibitory concentrations (MICs) of colistin against all 95 isolates of K. pneumoniae and E. coli strains in the LD colistin group were lower than $0.5 \mu \mathrm{g} / \mathrm{mL}$.

The overall 30-day mortality was significantly higher in patients receiving definitive therapy with LD colistin compared to carbapenems $(51.58 \%$ and $25.42 \%$, respectively, $P=0.001)$. A multivariate model indicated significant association between LD colistin and mortality as compared with carbapenems (HR 7.97; 95\% CI 3.68 to $17.25 ; P=0.001$ ) after adjustment for gender, ICU admission, duration of hospitalization, appropriate antibiotic treatment, Charlson comorbidity score, cerebrovascular disease, diabetes mellitus, infection type and nephrotoxicity. LD colistin was also independently associated with clinical failure (HR 4.30; 95\% CI 1.93 to 9.57; $P=0.001$ ) and bacteriologic failure (HR 9.49; 95\% CI 3.76 to 23.96; $P=0.001$ ) when adjusted for gender, ICU admission, use mechanical ventilation, duration of hospitalization, appropriate antibiotic treatment, Charlson comorbidity score, cerebrovascular disease, diabetes mellitus, infection type (i.e. pneumonia, urinary tract infection, bloodstream infection, skin and soft-tissue infections, infection at surgical sites, infection at joints and bones, and intra-abdominal infection) and nephrotoxicity (Table 2). 


\begin{tabular}{|c|c|c|c|c|c|c|}
\hline \multirow[b]{2}{*}{ Outcome parameter } & \multicolumn{2}{|c|}{$\begin{array}{l}\text { No. of patients (\%) with each outcome with } \\
\text { indicated treatment }\end{array}$} & \multirow{2}{*}{$\begin{array}{l}\text { Unadjusted HR (95\% } \\
\text { CI) }\end{array}$} & \multirow[b]{2}{*}{$p$ value } & \multirow{2}{*}{$\begin{array}{l}\text { Adjusted } \text { HR }^{*} \\
(95 \% \mathrm{CI})\end{array}$} & \multirow[b]{2}{*}{$p$ value } \\
\hline & LD colistin $(\mathrm{n}=95)$ & carbapenems $(n=301)$ & & & & \\
\hline 30-day mortality & $49(51.58)$ & $76(25.42)$ & $2.94(2.05-4.22)$ & 0.001 & $7.97(3.68-17.25)$ & 0.001 \\
\hline Clinical failure & $38(40.00)$ & $83(27.57)$ & $2.14(1.45-3.14)$ & 0.001 & $4.30(1.93-9.57)$ & 0.001 \\
\hline Bacteriological failure & $30(31.58)$ & $64(21.26)$ & $2.15(1.39-3.33)$ & 0.001 & $9.49(3.76-23.96)$ & 0.001 \\
\hline
\end{tabular}

Table 2. Cox regression analysis of outcomes of patients treated with LD colistin and carbapenems for the infection caused by ESBL-producing E. coli and K. pneumoniae. ${ }^{*}$ Adjusted for gender, ICU admission, use mechanical ventilation, duration of hospitalization, appropriate antibiotic treatment, Charlson comorbidity score, cerebrovascular disease, diabetes mellitus, infection type and nephrotoxicity. CI, confidence interval; HR hazard ratio.

\begin{tabular}{|c|c|c|c|c|c|}
\hline Variable & $\begin{array}{l}\text { No. of patients (\%) with } 30 \text {-day } \\
\text { mortality }\end{array}$ & Unadjusted HR (95\% CI) & $p$ value & $\begin{array}{l}\text { Adjusted HR* } \\
(95 \% \text { CI })\end{array}$ & $p$ value \\
\hline Meropenem $(n=241)$ & $60(24.90)$ & 1.00 (Reference) & & 1.00 (Reference) & \\
\hline LD colistin $(n=96)$ & $49(51.58)$ & $3.05(2.09-4.45)$ & 0.001 & $6.28(2.96-13.29)$ & 0.001 \\
\hline Ertapenem $(n=40)$ & $12(30.00)$ & 1.00 (Reference) & & 1.00 (Reference) & \\
\hline LD colistin $(n=96)$ & $49(51.58)$ & $2.43(1.23-4.82)$ & 0.011 & $7.58(2.85-20.14)$ & 0.001 \\
\hline Imipenem $(n=20)$ & $6(30.00)$ & 1.00 (Reference) & & 1.00 (Reference) & \\
\hline LD colistin $(n=96)$ & $49(51.58)$ & $2.77(1.10-6.99)$ & 0.030 & $7.03(2.15-23.03)$ & 0.001 \\
\hline
\end{tabular}

Table 3. Cox regression analysis of 30-day mortality of patients treated with LD colistin and carbapenems (meropenem, ertapenem, imipenem) for the infection caused by ESBL-producing E. coli and K. pneumoniae. * Adjusted for gender, ICU admission, use mechanical ventilation, duration of hospitalization, appropriate antibiotic treatment, Charlson comorbidity score, cerebrovascular disease, diabetes mellitus, infection type and nephrotoxicity. CI, confidence interval; HR hazard ratio.

\begin{tabular}{|c|c|c|c|c|c|}
\hline Infection types & $\begin{array}{l}\text { No. of patients (\%) with 30-day } \\
\text { mortality }\end{array}$ & Unadjusted HR (95\% CI) & $p$ value & $\begin{array}{l}\text { Adjusted HR* } \\
(95 \% \mathrm{CI})\end{array}$ & $p$ value \\
\hline \multicolumn{6}{|l|}{ Pneumonia } \\
\hline Carbapenems $(n=116)$ & $46(39.66)$ & 1.00 (Reference) & & 1.00 (Reference) & \\
\hline LD colistin $(\mathrm{n}=45)$ & $22(48.89)$ & $1.52(0.91-2.54)$ & 0.107 & $8.38(2.73-25.72)$ & 0.001 \\
\hline \multicolumn{6}{|l|}{ Urinary tract infection } \\
\hline Carbapenems $(n=165)$ & $31(18.79)$ & 1.00 (Reference) & & 1.00 (Reference) & \\
\hline LD colistin $(\mathrm{n}=31)$ & $17(54.84)$ & $4.43(2.43-8.07)$ & 0.001 & $6.64(1.80-24.58)$ & 0.005 \\
\hline \multicolumn{6}{|l|}{ Bloodstream infection } \\
\hline Carbapenems $(n=13)$ & $4(30.77)$ & 1.00 (Reference) & & 1.00 (Reference) & \\
\hline LD colistin $(n=41)$ & $23(56.10)$ & $3.17(1.07-9.40)$ & 0.038 & $9.51(1.48-60.97)$ & 0.018 \\
\hline
\end{tabular}

Table 4. Cox regression analysis of 30-day mortality of patients treated with LD colistin and carbapenems for the infection types caused by ESBL-producing E. coli and K. pneumoniae. * Adjusted for gender, ICU admission, use mechanical ventilation, duration of hospitalization, appropriate antibiotic treatment, Charlson comorbidity score, cerebrovascular disease, diabetes mellitus, infection type and nephrotoxicity. CI, confidence interval; HR hazard ratio.

The overall 30-day mortality rates were still higher in patients receiving definitive therapy with LD colistin compared to carbapenems, after subgroup analysis with type of carbapenems, i.e. meropenem, ertapenem and imipenem (Table 3), and with the infection type (Table 4).

Univariate cox-regression analysis showed that definitive therapy with LD colistin and ICU status were associated with increased mortality. In the multivariable analysis carried out with Cox regression, only definitive therapy with LD colistin showed association with mortality. However, underlying disease was also independently associated with clinical failure (Table 5). Mortality rates were significantly higher in patients receiving definitive therapy with LD colistin as compared to carbapenems $(P<0.001$ by log-rank test) (Fig. 1). 


\begin{tabular}{|c|c|c|c|c|c|c|}
\hline \multirow[b]{2}{*}{ Variable } & \multicolumn{3}{|c|}{ Univariable analysis } & \multicolumn{3}{|c|}{ Multivariable analysis } \\
\hline & Unadjusted HR & HR $(95 \% \mathrm{CI})$ & p-value & Adjust HR & HR $(95 \% \mathrm{CI})$ & p-value \\
\hline \multicolumn{7}{|l|}{ 30-day mortality } \\
\hline Definitive therapy with LD colistin & 2.94 & $2.05-4.22$ & 0.001 & 3.14 & $1.75-5.62$ & 0.001 \\
\hline ICU status & 1.91 & $1.33-2.73$ & 0.001 & 1.05 & $0.66-1.66$ & 0.838 \\
\hline Charlson score $\geq 4$ & 0.91 & $0.64-1.30$ & 0.621 & 1.13 & $0.77-1.65$ & 0.546 \\
\hline Age $\geq 60$ & 1.47 & $0.99-2.18$ & 0.054 & 1.49 & $0.99-2.23$ & 0.054 \\
\hline Underlying disease & 1.49 & $0.80-2.78$ & 0.203 & 1.51 & $0.80-2.85$ & 0.206 \\
\hline Nephrotoxicity & 2.48 & $1.60-3.82$ & 0.001 & 0.10 & $0.56-1.77$ & 0.996 \\
\hline \multicolumn{7}{|l|}{ Clinical failure } \\
\hline Definitive therapy with LD colistin & 2.14 & $1.45-3.15$ & 0.001 & 2.20 & $1.16-4.16$ & 0.016 \\
\hline ICU status & 1.28 & $0.87-1.88$ & 0.205 & 0.82 & $0.50-1.33$ & 0.432 \\
\hline Charlson score $\geq 4$ & 0.75 & $0.52-1.07$ & 0.117 & 0.83 & $0.56-1.21$ & 0.336 \\
\hline Age $\geq 60$ & 1.09 & $0.75-1.59$ & 0.641 & 1.02 & $0.69-1.50$ & 0.905 \\
\hline Underlying disease & 2.13 & $1.04-4.36$ & 0.039 & 2.35 & $1.13-4.91$ & 0.022 \\
\hline Nephrotoxicity & 2.20 & $1.38-3.49$ & 0.001 & 1.16 & $0.61-2.22$ & 0.654 \\
\hline \multicolumn{7}{|l|}{ Bacteriological failure } \\
\hline Definitive therapy with LD colistin & 2.16 & $1.39-3.33$ & 0.001 & 2.76 & $1.38-5.50$ & 0.004 \\
\hline ICU status & 1.30 & $0.84-2.00$ & 0.233 & 0.80 & $0.46-1.40$ & 0.445 \\
\hline Charlson score $\geq 4$ & 0.83 & $0.55-1.25$ & 0.378 & 0.93 & $0.61-1.44$ & 0.760 \\
\hline Age $\geq 60$ & 1.04 & $0.68-1.60$ & 0.842 & 0.97 & $0.63-1.50$ & 0.885 \\
\hline Underlying disease & 1.88 & $0.87-4.07$ & 0.107 & 2.07 & $0.95-4.63$ & 0.066 \\
\hline Nephrotoxicity & 1.82 & $1.05-3.18$ & 0.033 & 0.84 & $0.41-1.72$ & 0.626 \\
\hline
\end{tabular}

Table 5. Cox regression analysis of associations between different variables and primary outcomes including 30-day mortality, clinical response and bacteriological response. CI, confidence interval; HR hazard ratio.

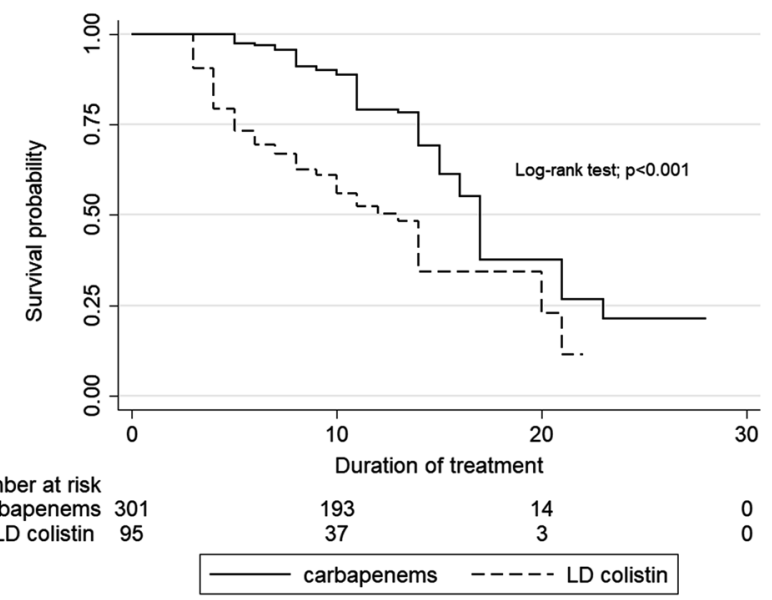

Figure 1. Kaplan-Meier curve showing survival probability of infection caused by ESBL-producing E. coli and K. pneumoniae according to LD colistin and carbapenems regimens.

\section{Discussion}

The clinical studies of colistin for the treatment of ESBL-producing Escherichia coli and Klebsiella pneumoniae are limited. This study was to evaluate clinical efficacy of loading dose (LD) colistin compared with carbapenems for treatment ESBL-producing Escherichia coli and Klebsiella pneumoniae. The unadjusted 30-day mortality was higher among LD colistin-treated patients than carbapenems-treated patients. The adjusted analysis showed higher mortality in the LD colistin group that was statistically significant in patients with ESBL-producing $E$. coli and K. pneumoniae. LD colistin was also independently associated with clinical failure and bacteriological failure. Based on these results, colistin was less effective than carbapenems. Thus, colistin should be reserved only for treatment of infections that are resistant to all antibiotics including carbapenems.

There are no strict guidelines or policies pertaining to the preferred therapeutic management of infections due to ESBL-producing Enterobacteriaceae although many health professionals have considered that carbapenems are the preferred agents ${ }^{11}$. Harris, et al. conducted a non-inferiority randomized clinical trial that included 
391 patients with ESBL-producing Enterobacteriaceae infection. They reported that definitive treatment with piperacillin-tazobactam did not provide non-inferior 30-day mortality as compared to meropenem. These findings therefore did not support use of piperacillin-tazobactam in this condition ${ }^{12}$.

Colistin has promising in-vitro activity against ESBL-producing Enterobacteriaceae including E. coli, K. pneumoniae and Enterobacter cloacae ${ }^{13}$. Despite it has the in vitro activity, the use of colistin in ESBL-producing Enterobacteriaceae treatment is still debatable because of limited available evidences in human trials ${ }^{13}$.

Our study found that after controlling for confounding factors, LD colistin was associated with increased risk of death (HR 3.14; 95\% CI 1.75 to $5.62 ; P<0.001$ ). Carbapenems were associated with significantly better clinical outcomes than the other antimicrobial, i.e. LD colistin. Moreover, the results from subgroup analysis with the infection type and type of carbapenems, found better survival rates of carbapenems in the subgroup analysis with infection types. This was similar to the findings from a study in patients with non-urinary ESBL bloodstream infections during 2010 and 2012. The multivariate analysis results showed that 10 patients who received piperacillin-tazobactam had higher 90 -day mortality as compared to 69 patients who received a carbapenem (adjusted odds ratio, 7.9, $P=0.03)^{14}$. Likewise, a randomized study indicated that $100 \%(10 / 10)$ of the patients with nosocomial pneumonia who were treated with imipenem had clinical response while only $69 \%(9 / 13)$ of patients treated with cefepime had the same outcome ${ }^{15}$.

Our results might be explained by three reasons. Firstly, rapid emergence of resistance of colistin monotherapy has been reported in vitro using pharmacokinetic/pharmacodynamic models to mimic the pharmacokinetics of colistin in patients ${ }^{16}$. Moreover, several reports indicated that use of colistin for ESBL-producing Klebsiella pneumoniae infection could lead to an emergence of colistin resistance in Klebsiella pneumoniae ${ }^{13,17}$. There are several mechanisms dependent on the bacterial species, e.g. the mobile colistin resistance genes $(m c r)$ in E. coli or the chromosomal mutations in regulatory genes of LPS synthesis in K. pneumonia ${ }^{18,19}$. However, the emergence of colistin-resistant bacteria was not observed in the present study. Secondly, when given colistin systemically, this drug is unlikely to be effective for pneumonia because of its poor penetration into the pulmonary parenchyma ${ }^{20}$. In the present study, $47.37 \%$ of pneumonia patients were in the colistin group. Thirdly, the variation of LD colistin concentrations was high; it was found that patients who received the same loading doses of CBA (300 mg) had different colistin concentrations at a steady state ${ }^{21}$. Therefore, patients in this study might have different concentrations of colistin, resulting in different bacterial effects.

Nephrotoxicity is the main adverse effect reported with the use of colistin. In the present study, nephrotoxicity was found in $48.42 \%$ of the patients in LD colistin group but was not found in carbapenem group $(P=0.001)$. However, using multivariate Cox-regression analysis, nephrotoxicity was not associated with 30-day mortality (HR 0.10 ; $95 \%$ CI 0.56 to $1.77 ; P=0.996$ ), clinical failure (HR 1.16 ; $95 \%$ CI 0.61 to $2.22 ; P=0.654$ ), and bacteriological failure (HR $0.84 ; 95 \%$ CI 0.41 to $1.72 ; P=0.626$ ).

The present study has some limitations. Firstly, this study was the difference in baseline characteristics. This difference, however, was found in most retrospective studies. Although we attempted to adjust for potential confounders in the statistical methods, residual unknown confounding factors could remain with this study design. Furthermore, a complete compensation of the basic differences is probably not possible and the adjusted hazard ratios are subject to high uncertainty. So, the results should be interpreted with caution due to possible confounders and lack of some information.

Secondly, colistin should be reserved for specific situations which no other drugs can be used. Therefore, randomized clinical trial is not an appropriate study design for this purpose. However, this large retrospective study provides evidence regarding the efficacy of colistin in the treatment of ESBL-producing Enterobacteriaceae. So, until more definitive studies are performed, our findings suggested that LD colistin was inferior to carbapenem therapy for the treatment of ESBL-associated infections.

Thirdly, the appropriate methods for determination of colistin (polymyxin E) MIC have been discussed for several years. EUCAST and CLSI recently published a joint recommendation that broth microdilution (BMD) is the only validated method for antimicrobial susceptibility testing of colistin. Other testing methods such as agar dilution, disk diffusion and gradient diffusion are not currently recommended. However, the use of broth microdilution method for susceptibility testing may not be practical in routine diagnostic microbiology laboratories because of the individual laboratory workloads. Therefore, many laboratories still use alternative methods such as gradient strips and semi-automated systems in order to manage their workloads ${ }^{22}$.

Chiang Mai University Hospital had used Vitek-2 semi-automated system (bioMérieux) to test susceptibility since 2005 because Vitek-2 was, at the time, reported as an acceptable testing method for colistin ${ }^{23-25}$. Moreover, for K. pneumoniae and E. coli, the Vitek-2 provided better performance for isolates with MIC $\leq 0.5$ and $\geq 16 \mu \mathrm{g} /$ $\mathrm{mL}$ than the isolates with MIC within $>0.5$ and $<16 \mu \mathrm{g} / \mathrm{mL}$. These results were presented by Girardello et al. ${ }^{26}$ that compared performance of Vitek-2 with BMD in determination of colistin susceptibility. The authors ${ }^{26}$ reported a good correlation of results between Vitek-2 and BMD when tested K. pneumoniae and E. coli with isolates of MICs $\leq 0.5$ and $\geq 16 \mu \mathrm{g} / \mathrm{mL}$. In addition, the Vitek- 2 was able to detect the resistance of 10 isolates of $m c r$-1-carrying E. coli although this polymyxin resistance mechanism exhibited borderline MICs of $4 \mu \mathrm{g} / \mathrm{mL}[6$ isolates] and $8 \mu \mathrm{g} / \mathrm{mL}$ [ 4 isolates]. Therefore, Girardello et al. suggested that susceptibility testing with the reference method (broth microdilution) might be unnecessary when Vitek-2-determined MICs were either very low $(\leq 0.5 \mu \mathrm{g} / \mathrm{mL})$ or very high $(\geq 16 \mu \mathrm{g} / \mathrm{mL})^{26}$.

Consistently, Lo-Ten-Foe et al. ${ }^{25}$ compared the Vitek-2 colistin susceptibility test to the BMD reference test and showed a high level of agreement; there were only heteroresistant E. cloacae isolates which Vitek-2 failed to detect. Additionally, the study by Lellouche et al. ${ }^{22}$, which was performed in 274 isolates with colistin MIC $\leq 1 \mu \mathrm{g} /$ $\mathrm{mL}$ by BMD, reported very good correlation between Vitek- 2 testing and BMD. However, for the isolates with MIC $>1 \mu \mathrm{g} / \mathrm{mL}$, Vitek-2 had poor correlation with the BMD as Vitek-2 yielded lower MICs.

With regards to the present study, the MICs of colistin against all 95 strains of ESBL-producing K. pneumoniae and E. coli in the LD colistin group were lower than $0.5 \mu \mathrm{g} / \mathrm{mL}$. Therefore, the susceptibility of colistin in 
the present study was very likely to be acceptably reliable according to the above-mentioned results. Moreover, our study included only ESBL-producing K. pneumoniae and E. coli, but did not include carbapenem-resistant Enterobacteriaceae (CRE). It is known that MICs of ESBL-producing K. pneumoniae and E. coli were usually less than the MICs of CRE, so we were convinced that all isolates in our study were really sensitive to carbapenem.

Based on the information provided, ESBL infections in present study should not be misclassified as falsesusceptible for colistin and treated with colistin.

\section{Conclusions}

LD colistin treatment was associated with poorer survival rate compared to carbapenems. Adjusted analyses also suggested that LD colistin was less effective than carbapenems. Thus, patients who were infected with ESBLproducing Enterobacteriaceae should be firstly treated with carbapenems. LD colistin should be considered as an alternative to carbapenems for treatment of ESBL-producing Enterobacteriaceae only in the circumstances where carbapenems cannot be used such as infections with carbapenem-resistant Gram-negative pathogens, patients with history of type 1 hypersensitivity of penicillin, or allergic reaction to penicillin was severe (e.g. anaphylactic shock). However, the conclusion in this study was based on only retrospective data with differences in baseline characteristics. Although hazard ratio adjustment to compensate these differences was well performed, obviously it was not possible to completely remove all confounders. Therefore, interpreting the results from our study should be done very carefully.

\section{Methods}

Study setting and participants. A retrospective cohort study from 1 January 2005 to 30 June 2015 pertaining to outcomes of ESBL-producing E. coli and K. pneumoniae was conducted at the Chiang Mai University Hospital in Chiang Mai University. This study was approved by the ethics committee on human research of the Faculty of Medicine, Chiang Mai University of a waiver of informed consent for retrospective data collection under the condition of anonymously stored data collected. All methods were performed in accordance with the relevant guidelines and regulations. Patients were included if they were equal or greater than 18 years old and had a microbiologically documented infection with ESBL-producing E. coli or K. pneumoniae infection. Infection was defined according to the Center for Disease Control and Prevention (CDC) criteria ${ }^{27}$. Patients were excluded if they had received $<2$ doses of the studied drug, i.e. LD colistin and carbapenems, received any other agents with activity against the offending ESBL isolate (e.g. aminoglycosides, fluoroquinolones, trimethoprim/ sulfamethoxazole and fosfomycin), had other types of Gram-negative infections, received hemodialysis or renal replacement therapy. The recruited patients with ESBL infection were divided into two groups of LD colistin and carbapenems.

The carbapenems case was defined as a patient who had received any carbapenems, e.g. ertapenem, imipenem and meropenem in order to treat the documented ESBL infection for longer than $48 \mathrm{~h}$ and received only one course the treatment. Likewise, LD colistin case was defined as a patient who had been treated the documented ESBL infection with $300 \mathrm{mg}$ of colistin base activity (CBA) once at the start of treatment course for longer than $48 \mathrm{~h}$ and treated only one course of colistin. The definitive therapy included patients receiving definitive monotherapy with LD colistin or carbapenems (imipenem/cilastatin, meropenem, ertapenem) for treatment of the infection with the ESBL-producing organism.

Dosage regimens of antibiotic were usually based on the respective hospital guidelines: LD colistin $300 \mathrm{mg}$ of CBA once at the start of treatment course, and then $150 \mathrm{mg}$ of CBA every $12 \mathrm{~h}$, meropenem $1 \mathrm{~g}$ every $8 \mathrm{~h}$, imipenem $500 \mathrm{mg}$ every $6 \mathrm{~h}$ and ertapenem $1 \mathrm{~g}$ every $24 \mathrm{~h}$. All doses were adjusted for renal function accordingly.

Data collection. Patient data were collected through computerized medical records and patient chart review. The following data were obtained from medical records: age, gender, intensive care unit (ICU) admission during infection, Charlson score, underlying disease, source of infection (as documented in the medical record by the treating physicians), duration of positive cultures and results of antimicrobial susceptibility testing, length of stay, invasive mechanical ventilation, timing of antibiotic therapy, mortality status and nephrotoxicity.

Outcome assessment. Three primary outcomes in this study were mortality rate at 30 days, clinical and bacteriological responses after the start of treatment. Thirty-day mortality was defined as death within 30 days of an ESBL-producing E. coli or K. pneumoniae infection. Clinical response of treatment was assessed by resolution or partial resolution of fever, leukocytosis, and local signs and symptoms of ESBL infections at the end of treatment. Clinical failure was defined as failure to meet all criteria for clinical response. Microbiological response was defined as obtaining two consecutive negative ESBL cultures from the site of infection after the initial positive culture, whereas microbiological failure was defined as persistence of the original causative organism in the subsequent specimen cultures. Nephrotoxicity was counted if patients developed any grades of renal failure based on RIFLE criteria.

Statistical analysis. All statistical analyses were carried out using Stata software, version 14 (Stata-Corp, College Station, TX). Descriptive statistics were used to describe the data: frequencies and percentages for categorical variables and means with standard deviations for continuous variables. To compare two groups, Fisher's exact test was used for categorical variables and independent t-test was used for continuous variables. A twotailed test result with $P$ value of $<0.05$ was considered to be statistically significant. The rate of time to 30 -day mortality, clinical failure and microbiological failure were evaluated using Cox regression model that controlled different variables between the two groups. Hazard ratios (HRs) were reported, with 95\% confidence intervals (CIs). Potential confounders and interactions were added using a backward method. Additional adjustment for 
any variables with a $P$ value $<0.20$ on univariable analysis were included in an adjusted Cox regression model. In addition, all variables that demonstrated a trend toward association with outcomes were forced into the model at the discretion of the investigator and statistical significance was set at $P \leq 0.05$. The proportional hazard assumption was checked for all models. Mortality rates of patients treated with LD colistin or carbapenems were compared using Kaplan-Meier curve and log-rank test. All tests were 2-tailed and $P$ values $\leq 0.05$ were used for statistical significance testing.

Antimicrobial susceptibility testing. E. coli and K. pneumoniae were identified at a division of Clinical Microbiology, CMUH, using conventional cultures and biochemical methods. Antibiotic susceptibility was performed by disk diffusion method and minimal inhibitory concentrations (MICs) using E-test, encompassing nearly all important antibiotics ${ }^{28}$. The presence of ESBL was detected using the double-disc synergy test between clavulanate and third-generation cephalosporins (i.e. cefotaxime, cefpodoxime, ceftriaxone and ceftazidime). Antibiotic susceptibility and MICs were analyzed according to the CLSI ${ }^{28}$. Colistin susceptibility was determined by Vitek-2 automated method (bioMérieux, Marcy-L'Etoile, France) ${ }^{23-26}$. This study used colistin susceptibility $(\leq 2 \mu \mathrm{g} / \mathrm{mL})$ following the European Committee on Antimicrobial Susceptibility Testing (EUCAST) breakpoints $^{29}$.

Ethics approval and consent to participate. This retrospective cohort study was approved by the ethics committee on human research of the Faculty of Medicine, Chiang Mai University. (NONE-2558-02791).

\section{Data availability}

The datasets used and analyzed during the current study are available from the corresponding author on reasonable request.

Received: 28 May 2020; Accepted: 17 November 2020

Published online: 08 January 2021

\section{References}

1. Sawatwong, P. et al. High burden of extended-spectrum $\beta$-lactamase-producing Escherichia coli and Klebsiella pneumoniae bacteremia in older adults: A seven-year study in two rural Thai provinces. Am. J. Trop. Med. Hyg. 100, 943-951 (2019).

2. Tamma, P. D. \& Rodriguez-Bano, J. The use of noncarbapenems $\beta$-lactams for the treatment of extended-spectrum $\beta$-lactamase infections. Clin. Infect Dis. 64, 972-980 (2017).

3. Sheu, C. C. et al. Management of infections caused by extended-spectrum $\beta$-lactamase-producing Enterobacteriaceae: Current evidence and future prospects. Expert. Rev. Anti. Infect. Ther. 16, 205-218 (2018).

4. Paterson, D. L. et al. Antibiotic therapy for Klebsiella pneumoniae bacteremia: Implications of production of extended-spectrum beta-lactamases. Clin. Infect. Dis. 39, 31-37 (2004).

5. Vardakas, K. Z., Tansarli, G. S., Rafailidis, P. I. \& Falagas, M. E. Carbapenems versus alternative antibiotics for the treatment of bacteraemia due to Enterobacteriaceae producing extended-spectrum $\beta$-lactamases: A systematic review and meta-analysis. J. Antimicrob. Chemother. 67, 2793-2803 (2012).

6. Li, J., Nation, R. L., Milne, R. W., Turnidge, J. D. \& Coulthard, K. Evaluation of colistin as an agent against multi-resistant Gramnegative bacteria. Int. J. Antimicrob. Agents 25, 11-25 (2005).

7. Nation, R. L. \& Li, J. Colistin in the 21 st century. Curr. Opin. Infect. Dis. 22, 535-543 (2009).

8. Bishara, J. et al. Antibacterial susceptibility of extended-spectrum beta-lactamase-producing Klebsiella pneumoniae and Escherichia coli. Isr. Med. Assoc. J. 7, 298-301 (2005).

9. Parchuri, S., Mohan, S. \& Cunha, B. A. Extended spectrum $\beta$-lactamase-producing Klebsiella pneumoniae chronic ambulatory peritoneal dialysis peritonitis treated successfully with polymyxin B. Heart Lung. 34, 360-363 (2005).

10. Segal-Maurer, S., Mariano, N., Qavi, A., Urban, C. \& Rahal, J. J. Successful treatment of ceftazidime-resistant Klebsiella pneumoniae ventriculitis with intravenous meropenem and intraventricular polymyxin B: Case report and review. Clin. Infect. Dis. 28, 1134-1138 (1999).

11. Paterson, D. L. \& Bonomo, R. A. Extended-spectrum $\beta$-lactamases: A clinical update. Clin. Microbiol. Rev. 18, 657-686 (2005).

12. Harris, P. N. A. et al. Effect of piperacillin-tazobactam vs meropenem on 30-day mortality for patients with E. coli or Klebsiella pneumoniae bloodstream infection and ceftriaxone resistance: A randomized clinical trial. JAMA 320, 984-994 (2018),

13. Ku, Y. H., Lee, M. F., Chuang, Y. C., Chen, C. C. \& Yu, W. L. In vitro activity of colistin sulfate against Enterobacteriaceae producing extended-spectrum $\beta$-lactamases. J. Microbiol. Immunol. Infect. 48, 699-702 (2015).

14. Ofer-Friedman, H. et al. Carbapenems versus piperacillin-tazobactam for bloodstream infections of nonurinary source caused by extended-spectrum beta-lactamase producing Enterobacteriaceae. Infect. Control Hosp. Epidemiol. 36, 981-985. https://doi. org/10.1017/ice.2015.101 (2015)

15. Zanetti, G. et al. Cefepime versus imipenem-cilastatin for treatment of nosocomial pneumonia in intensive care unit patients: A multicenter, evaluator-blind, prospective, randomized study. Antimicrob. Agents Chemother. 47, 3442-3447 (2003).

16. Bergen, P. J. et al. Comparison of once-, twice- and thrice-daily dosing of colistin on antibacterial effect and emergence of resistance: Studies with Pseudomonas aeruginosa in an in vitro pharmacodynamic model. J. Antimicrob. Chemother. 61, 636-642 (2008).

17. Neonakis, I. K. et al. Resistance status and evolution trends of Klebsiella pneumoniae isolates in a university hospital in Greece: Ineffectiveness of carbapenems and increasing resistance to colistin. Chemotherapy 56, 448-452 (2010).

18. Liu, Y. Y. et al. Emergence of plasmid-mediated colistin resistance mechanism MCR-1 in animals and human beings in China: A microbiological and molecular biological study. Lancet Infect. Dis. 16, 161-168. https://doi.org/10.1016/S1473-3099(15)00424-7 (2016).

19. Olaitan, A. O., Morand, S. \& Rolain, J. M. Mechanisms of polymyxin resistance: Acquired and intrinsic resistance in bacteria. Front. Microbiol. 5, 643. https://doi.org/10.3389/fmicb.2014.00643 (2014).

20. Imberti, R. et al. Steady-state pharmacokinetics and BAL concentration of colistin in critically Ill patients after IV colistin methanesulfonate administration. Chest 138, 1333-1339 (2010).

21. Karaiskos, I. et al. Colistin population pharmacokinetics after application of a loading dose of $9 \mathrm{MU}$ colistin methanesulfonate in critically Ill patients. Antimicrob. Agents Chemother. 59, 7240-7248 (2015).

22. Lellouche, J. et al. AIDA study group. Combining VITEK 2 with colistin agar dilution screening assist timely reporting of colistin susceptibility. Clin. Microbiol. Infect. 25, 711-716. https://doi.org/10.1016/j.cmi.2018.09.014 (2019). 
23. Dafopoulou, K. et al. Comparative evaluation of colistin susceptibility testing methods among carbapenem-nonsusceptible Klebsiella pneumoniae and Acinetobacter baumannii clinical isolates. Antimicrob. Agents Chemother. 59, 4625-4630. https://doi. org/10.1128/AAC.00868-15 (2015).

24. Lee, S. Y. et al. Comparison of the 2, MicroScan, and Etest methods with the agar dilution method in assessing colistin susceptibility of bloodstream isolates of Acinetobacter species from a Korean University Hospital. J. Clin. Microbiol. 51, 1924-1926. https://doi. org/10.1128/JCM.00427-13 (2013).

25. Lo-Ten-Foe, J. R. et al. Comparative evaluation of the Vitek 2, disk diffusion, Etest, broth microdilution, and agar dilution susceptibility testing methods for colistin in clinical isolates, including heteroresistant Enterobacter cloacae and Acinetobacter baumannii strains. Antimicrob. Agents Chemother. 51, 3726-3730. https://doi.org/10.1128/AAC.01406-06 (2007).

26. Girardello, R. et al. Colistin susceptibility testing and Vitek-2: Is it really useless?. Diagn. Microbiol. Infect. Dis. 91, 309-311 (2018).

27. Horan, T. C., Andrus, M. \& Dudeck, M. A. CDC/NHSN surveillance definition of health care-associated infection and criteria for specific types of infections in the acute care setting. Am. J. Infect. Control 36, 309-332 (2008).

28. Clinical and Laboratory Standards Institute. Performance standards for antimicrobial susceptibility testing. Nineteenth informational supplement. Approved standard M100-S19. Wayne, PA: CLSI (2009).

29. The European Committee on Antimicrobial Susceptibility Testing. Breakpoint tables for interpretation of MICs and zone diameters. Version 8.0, Preprint at http://www.eucast.org. (2018)

\section{Acknowledgements}

This research work was partially supported by Chiang Mai University.

\section{Author contributions}

Conceptualization, W.K.; Data curation, W.K.,J.Y.; Formal analysis, W.K.; Investigation, W.K.; Methodology, W.K.; Project administration, W.K.; Software, W.K.; Supervision, P.O.; Validation, J.Y., S.O. and P.O.; Writing—original draft, W.K. and S.O.; Writing-review \& editing, W.K. and S.O.

\section{Competing interests}

The authors declare no competing interests.

\section{Additional information}

Correspondence and requests for materials should be addressed to W.K.

Reprints and permissions information is available at www.nature.com/reprints.

Publisher's note Springer Nature remains neutral with regard to jurisdictional claims in published maps and institutional affiliations.

(c) (1) Open Access This article is licensed under a Creative Commons Attribution 4.0 International License, which permits use, sharing, adaptation, distribution and reproduction in any medium or format, as long as you give appropriate credit to the original author(s) and the source, provide a link to the Creative Commons licence, and indicate if changes were made. The images or other third party material in this article are included in the article's Creative Commons licence, unless indicated otherwise in a credit line to the material. If material is not included in the article's Creative Commons licence and your intended use is not permitted by statutory regulation or exceeds the permitted use, you will need to obtain permission directly from the copyright holder. To view a copy of this licence, visit http://creativecommons.org/licenses/by/4.0/.

(c) The Author(s) 2021 